\title{
Tomography from collective measurements
}

\author{
A. Muñoz ${ }^{1,2} \cdot$ A. B. $\operatorname{Klimov}^{1}$ (D) M. Grass $^{3}$ (D) $\cdot$ L. L. Sánchez-Soto ${ }^{3,4}$ (D) \\ Received: 27 January 2018 / Accepted: 30 August 2018 / Published online: 14 September 2018 \\ (c) The Author(s) 2018
}

\begin{abstract}
We discuss the tomography of $N$-qubit states using collective measurements. The method is exact for symmetric states, whereas for not completely symmetric states the information accessible can be arranged as a mixture of irreducible SU(2) blocks. For the fully symmetric sector, the reconstruction protocol can be reduced to projections onto a canonically chosen set of pure states.
\end{abstract}

Keywords Quantum tomography · Quantum information · Collective measurements $\cdot$ Symmetric states

\section{Introduction}

Continuous-variable tomography has been exhaustively explored, from both theoretical and experimental viewpoints [1]. However, the corresponding problem for discrete systems stands as challenge [2]. If we look at the example of $N$ qubits, which will be our thread in this paper, one has to make at least $2^{N}+1$ measurements in different bases before to determine the state of an a priori unknown system [3-6]. With such an exponential scaling, it is clear that only few-qubit states can be reconstructed in a reasonable time $[7,8]$.

As a result, alternative techniques are called for. A wide class of new protocols are explicitly targeted for particular types of states. This includes states with low rank [9-12], such as matrix product states (MPS) [13,14], or multiscale entanglement renormalization ansatz (MERA) states [15]. The extra assumption of permutationally invariance was also examined [16-20], reducing the scaling of the required setups to $N^{3}$.

\footnotetext{
$凶 \quad$ L. L. Sánchez-Soto

1sanchez@fis.ucm.es

1 Departamento de Física, Universidad de Guadalajara, 44420 Guadalajara, Jalisco, Mexico

2 Departamento de Fisica, Universidad de Concepción, Casilla 160-C, Concepción, Chile

3 Max-Planck-Institut für die Physik des Lichts, Staudtstraße 2, 91058 Erlangen, Germany

4 Departamento de Óptica, Facultad de Física, Universidad Complutense, 28040 Madrid, Spain
} 
In the same spirit of simplicity, one may be tempted to examine the case when one can extract only partial information from the system under consideration. This happens, e.g., in large multipartite systems, wherein addressing individual particles turns out to be a formidable task. Bose-Einstein condensates constitute an archetype of this situation: only collective spin observables can be efficiently measured through detection of the spontaneous emission correlation functions [21,22].

By assessing collective spin operators, one can only access the SU(2) invariant subspaces appearing in the decomposition of the $N$-qubit density matrix. The problem of partial state tomography appears thus analogous to that of permutationally invariant states.

In the present work, we show that one can obtain an explicit partial reconstruction for the $N$-qubit density matrix in terms of average values of correlation functions of approximately $N^{3}$ collective spin operators. In other words, we propose to arrange $O\left(N^{3}\right)$ experimental data points inside SU(2) invariant subspaces. As an illustration, we analyze the fidelity of the reconstructed states for 2 and 3 qubits. In addition, we demonstrate that when the state belongs to the fully symmetric (Dicke) subspace, the tomographic measurements reduce to rank-one positive operator valued measurements (POVMs), and we find the corresponding operational expansion. As a bonus, we introduce a new type of discrete special functions that might find further applications in the analysis of $N$-qubit systems.

The paper is organized as follows. In Sect. 2 we briefly recall the principal aspects of discrete phase-space distribution functions and of the standard tomographic scheme. In Sect. 3 we provide explicit expressions for the permutationally invariant tomography for a $N$-qubit system, whereas in Sect. 4 an alternative scheme for fully symmetric states is presented. Finally, Sect. 5 summarizes our main results.

\section{Standard discrete tomography}

For a system of $N$ qubits, the Hilbert space is the tensor product $\mathbb{C}^{2} \otimes \cdots \otimes \mathbb{C}^{2}=\mathbb{C}^{2 N}$. The generators of the Pauli group $\mathscr{P}_{N}$ can be written as $[23,24]$

$$
\hat{Z}_{\alpha}=\hat{\sigma}_{z}^{\alpha_{1}} \otimes \cdots \otimes \hat{\sigma}_{z}^{\alpha_{N}}, \quad \hat{X}_{\beta}=\hat{\sigma}_{x}^{\beta_{1}} \otimes \cdots \otimes \hat{\sigma}_{x}^{\beta_{N}},
$$

so that they are labeled by $N$-tuples $\alpha=\left(\alpha_{1}, \ldots, \alpha_{N}\right)$ and $\beta=\left(\beta_{1}, \ldots, \beta_{N}\right)$, with $\alpha_{j}, \beta_{j} \in \mathbb{Z}_{2}$. Here, $\hat{\sigma}_{z}$ and $\hat{\sigma}_{x}$ are the usual Pauli operators on the $i$ th qubit: $\hat{\sigma}_{z}=$ $|0\rangle\langle 0|-| 1\rangle\langle 1|$ and $\hat{\sigma}_{x}=|0\rangle\langle 1|+| 1\rangle\langle 0|$ in the orthonormal computational basis $\{|0\rangle,|1\rangle\}$.

We next define the operators

$$
\hat{\Delta}^{(s)}(\alpha, \beta)=\frac{1}{2^{N(s+3) / 2}} \sum_{\gamma, \delta \in \mathbb{Z}_{2}^{N}}(-1)^{\alpha \delta+\beta \gamma+\gamma \delta(1-s) / 2}\left\langle\xi\left|\hat{Z}_{\gamma} \hat{X}_{\delta}\right| \xi\right\rangle^{-s} \hat{Z}_{\gamma} \hat{X}_{\delta}
$$


where $s= \pm 1$. From a physical perspective, the fiducial state $|\xi\rangle$ can be chosen as a factorized symmetric (with respect to particle permutations) state $|\xi\rangle=\otimes_{i=1}^{N}|\xi\rangle_{i}$

$$
|\xi\rangle_{i}=\frac{1}{\sqrt{1+|\xi|^{2}}}\left(|0\rangle_{i}+\xi|1\rangle_{i}\right)
$$

and $\xi=\frac{\sqrt{3}-1}{\sqrt{2}} e^{i \pi / 4}$, which corresponds to a spin coherent state determined by the normalized vector $\mathbf{n}=(1,1,1) / \sqrt{3}$ on the Bloch sphere [25]. The operators $\hat{\Delta}^{(s)}$ form a biorthogonal operator basis, namely

$$
\operatorname{Tr}\left[\hat{\Delta}^{(1)}\left(\alpha^{\prime}, \beta^{\prime}\right) \hat{\Delta}^{(-1)}(\alpha, \beta)\right]=2^{N} \delta_{\alpha \alpha^{\prime}} \delta_{\beta \beta^{\prime}}
$$

In complete analogy with the continuous case [26,27], any operator $\hat{A}$ acting on the Hilbert space $\mathbb{C}^{2^{N}}$ can be expanded in this basis as

$$
\hat{A}=\sum_{\alpha, \beta \in \mathbb{Z}_{2}^{N}} Q_{A}(\alpha, \beta) \hat{\Delta}^{(1)}(\alpha, \beta),
$$

where $Q_{A}(\alpha, \beta)=\operatorname{Tr}\left[\hat{A} \Delta^{(-1)}(\alpha, \beta)\right]$.

Actually, the kernel $\hat{\Delta}^{(-1)}(\alpha, \beta)$ can be represented as a rank-one projector

$$
\hat{\Delta}^{(-1)}(\alpha, \beta)=|\alpha, \beta\rangle\langle\alpha, \beta|
$$

Here, $|\alpha, \beta\rangle$ are discrete coherent states, constructed as [28-32]

$$
|\alpha, \beta\rangle=\exp [i \chi(\alpha, \beta)] \hat{Z}_{\alpha} \hat{X}_{\beta}|\xi\rangle
$$

where $\exp [i \chi(\alpha, \beta)]$ is an appropriately chosen phase that is irrelevant for our purposes here. Up to normalization, the set of projectors (6) forms an informationally complete POVM [33,34] with the choice (3) for the fiducial state. They satisfy the condition

$$
\sum_{\alpha, \beta \in \mathbb{Z}_{2}^{N}}|\alpha, \beta\rangle\langle\alpha, \beta|=2^{N} 1 .
$$

In the single-qubit case they form a SIC-POVM with four elements, and for $N$ qubits, they correspond to tensor products of the single-qubit SIC-POVM elements. Equation (5) can be thus interpreted as a tomographic reconstruction of the operator $\hat{A}$ in terms of measured probabilities $Q_{A}(\alpha, \beta)=\langle\alpha, \beta|\hat{A}| \alpha, \beta\rangle$. Moreover, $Q=\operatorname{Tr}\left[\hat{A} \Delta^{(-1)}\right]$ and $P=\operatorname{Tr}\left[\hat{A} \Delta^{(1)}\right]$ are discrete analogous of their continuous counterparts, defined in a $2^{N} \times 2^{N}$ discrete phase space $[35,36]$. 


\section{Tomography from collective measurements}

The representation (5) requires measuring the POVM (6) with $2^{2 N}$ elements. This provides a minimal complete tomography, but it is extremely demanding for $N \gg 1$.

As heralded in Sect. 1 to circumvent this problem we restrict ourselves to collective measurements. The information acquired from such measurements does not allow to obtain complete information about the state of the system: operators that are invariant under particle permutations (collective operators) "see" only irreducible subspaces appearing in the tensor decomposition of $\mathrm{SU}(2)^{\otimes N}$. Nonetheless, this still provides nontrivial information.

Symmetric operators $\hat{A}_{\text {sym }}$ on $N$ qubits are those invariant with respect to particle permutations:

$$
\hat{A}_{\mathrm{sym}}=\hat{\Pi}_{i j}^{\dagger} \hat{A}_{\mathrm{sym}} \hat{\Pi}_{i j} \quad \forall i, j=1, \ldots, N, \quad i \neq j,
$$

where $\hat{\Pi}_{i j}$ is the unitary operator that swaps particles $i$ and $j$. The crucial observation for what follows is that these operators possess a peculiar property: their symbols $P_{A_{\text {sym }}}(\alpha, \beta)$ depend exclusively on the Hamming weights [37] of $\alpha, \beta$, and their binary sum $\alpha+\beta$; that is,

$$
P_{A_{\mathrm{sym}}}(\alpha, \beta)=P_{A_{\mathrm{sym}}}(h(\alpha), h(\beta), h(\alpha+\beta)),
$$

with $h(\kappa)=\left|\left\{i: i=1, \ldots, N \mid \kappa_{i} \neq 0\right\}\right|, 0 \leq h(\kappa) \leq N$.

Therefore, the whole information about any symmetric measurement $\left\langle\hat{A}_{\text {sym }}\right\rangle=$ $\operatorname{Tr}\left(\hat{\rho} \hat{A}_{\text {sym }}\right)$ is conveniently conveyed in the projected $\tilde{Q}$-function [38,39],

$$
\tilde{Q}_{\rho}(m, n, k)=\sum_{\alpha, \beta \in \mathbb{Z}_{2}^{N}} Q_{\rho}(\alpha, \beta) \delta_{h(\alpha), m} \delta_{h(\beta), n} \delta_{h(\alpha+\beta), k},
$$

since, as it immediately follows from (5),

$$
\left\langle\hat{A}_{\mathrm{sym}}\right\rangle=\sum_{m, n=0}^{N} \sum_{k} P_{A_{\mathrm{sym}}}(m, n, k) \tilde{Q}_{\rho}(m, n, k),
$$

the index $k$ running in steps of two: $k=|m-n|,|m-n|+2, \ldots, \min (m+n, N, 2 N-$ $m-n)$.

If $\tilde{Q}_{\rho}(m, n, k)$ is available from measurements, one can lift it from the threedimensional $(m, n, k)$ space into the full $2^{N} \times 2^{N}$ discrete phase space according to

$$
Q_{\rho}^{\text {lifted }}(\alpha, \beta)=R_{m n k}^{-1} \sum_{m, n, k} \delta_{h(\alpha), m} \delta_{h(\beta), n} \delta_{h(\alpha+\beta), k} \tilde{Q}_{\rho}(m, n, k),
$$


where

$$
\begin{aligned}
R_{m n k} & =\sum_{\mu, \lambda \in \mathbb{Z}_{2}^{N}} \delta_{h(\mu), m} \delta_{h(\lambda), n} \delta_{h(\mu+\lambda), k} \\
& =\frac{N !}{\left(\frac{m+n-k}{2}\right) !\left(\frac{2 N-m-n-k}{2}\right) !\left(\frac{n-m+k}{2}\right) !\left(\frac{m-n+k}{2}\right) !}
\end{aligned}
$$

is a normalization factor fixed by the number of binary tuples $\lambda, \mu$ with Hamming weights $(h(\lambda), h(\mu), h(\lambda+\mu))=(m, n, k)$.

The reconstruction (13) of the $Q_{\rho}(\alpha, \beta)$ function from the projected one $\tilde{Q}_{\rho}(m, n, k)$ is incomplete; i.e., the map (11) is not faithful. The lifting (13) is thus just a way of organizing information obtained from $\left(\begin{array}{c}N+3 \\ 3\end{array}\right)=(N+1)(N+2)(N+3) / 6$ collective measurements, corresponding to the total number of possible triplets $(m, n, k)$ of Hamming weights, in a $2^{N} \times 2^{N}$ matrix.

By replacing $Q_{\rho}(\alpha, \beta)$ by $Q_{\rho}^{\text {lifted }}(\alpha, \beta)$ in the reconstruction (5), we get

$$
\hat{\rho}_{\mathrm{rec}}=\sum_{m, n, k} R_{m n k}^{-1} \tilde{Q}_{\rho}(m, n, k) \hat{\Delta}^{(1)}(m, n, k),
$$

where the symmetric operators $\hat{\Delta}^{( \pm 1)}(m, n, k)$ can be jotted down as

$$
\begin{aligned}
\hat{\Delta}^{( \pm 1)}(m, n, k) & =\sum_{\alpha, \beta \in \mathbb{Z}_{2}^{N}} \delta_{h(\alpha), m} \delta_{h(\beta), n} \delta_{h(\alpha+\beta), k} \hat{\Delta}^{( \pm 1)}(\alpha, \beta) \\
& =2^{-(3 \pm 1) N / 2} \sum_{m^{\prime}, n^{\prime}, k^{\prime}} g_{m n k}\left(m^{\prime}, n^{\prime}, k^{\prime}\right) 3^{ \pm\left(m^{\prime}+n^{\prime}+k^{\prime}\right) / 4} \hat{F}_{m^{\prime} n^{\prime} k^{\prime}}
\end{aligned}
$$

Here, $\hat{F}_{m n k}$ stands for the orthonormal set of operators (see Appendix A for details)

$$
\hat{F}_{m n k}=\sum_{\mu, \lambda \in Z_{2}^{N}} \delta_{h(\mu), m} \delta_{h(\lambda), n} \delta_{h(\mu+\lambda) k}(-i)^{\mu \lambda} \hat{Z}_{\mu} \hat{X}_{\lambda}
$$

and

$$
g_{m n k}(h(\alpha), h(\beta), h(\alpha+\beta))=\sum_{\gamma, \delta \in \mathbb{Z}_{2}^{N}}(-1)^{\alpha \delta+\beta \gamma} \delta_{h(\gamma), m} \delta_{h(\delta), n} \delta_{h(\gamma+\delta), k}
$$

are discrete functions, whose properties are explored in Appendix B.1. Observe that (18) is independent of the choice of $\alpha$ and $\beta$ : any other choice $\alpha^{\prime}$ and $\beta^{\prime}$ is related by permutations, which can be applied to $\gamma$ and $\delta$ as well.

Because of the properties of $\hat{F}_{m n k}$, the reconstruction (15) can be reduced to

$$
\hat{\rho}_{\text {rec }}=\frac{1}{2^{N}} \sum_{m, n, k} R_{m n k}^{-1}\left\langle\hat{F}_{m n k}\right\rangle \hat{F}_{m n k}
$$


which is an explicit function of $\left(\begin{array}{c}N+3 \\ 3\end{array}\right)$ expectation values of collective operators.

Note, in passing, that the operators (17) can be always expanded in terms of collective spin operators. For instance, by direct inspection one gets that in the simplest cases $n=0$ or $m=0, \hat{F}_{m n k}$ are diagonal in the computational basis:

$$
\begin{aligned}
& \hat{F}_{000}=11 \quad \hat{F}_{101}=\hat{S}_{z}, \quad \hat{F}_{202}=\frac{1}{2 !}\left(\hat{S}_{z}^{2}-N 1\right), \\
& \hat{F}_{303}=\frac{1}{3 !}\left[\hat{S}_{z}^{3}-(3 N-2) \hat{S}_{z}\right],
\end{aligned}
$$

where $\hat{S}_{j}=\sum_{i=1}^{N} \hat{\sigma}_{j}^{(i)}$.

By construction, $\hat{\rho}_{\text {rec }}$ is nonzero only inside $S U(2)$ invariant blocks and coincides with the true density matrix in the fully symmetric subspace. In all the other blocks, $\hat{\rho}_{\text {rec }}$ differs from the true value and, in particular, the irreducible subspaces of the same dimension are indistinguishable in $\hat{\rho}_{\text {rec }}$.

Let us illustrate the approach with a couple of basic examples. An arbitrary pure two-qubit state can be parametrized as

$$
|\psi\rangle=\sin \theta\left|\psi_{\mathrm{anti}}\right\rangle+e^{i \beta} \cos \theta\left|\psi_{\mathrm{sym}}\right\rangle
$$

where $\left|\psi_{\text {sym }}\right\rangle$ and $\left|\psi_{\text {anti }}\right\rangle$ denote states from the symmetric and antisymmetric subspaces correspondingly, represented in the computational basis as

$$
\begin{aligned}
\left|\psi_{\mathrm{anti}}\right\rangle= & \frac{|01\rangle-|10\rangle}{\sqrt{2}}, \\
\left|\psi_{\mathrm{sym}}\right\rangle= & \sin \alpha_{1}|00\rangle+e^{i \gamma_{1}} \cos \alpha_{1} \sin \alpha_{2}\left(\frac{|01\rangle+|10\rangle}{\sqrt{2}}\right) \\
& +e^{i \gamma_{2}} \cos \alpha_{1} \cos \alpha_{2}|11\rangle,
\end{aligned}
$$

$0 \leq \alpha_{1}, \alpha_{2} \leq \pi / 2,0 \leq \gamma_{1}, \gamma_{2}<2 \pi$. The reconstructed density matrix is the incoherent mixture

$$
\hat{\rho}_{\text {rec }}=\sin ^{2} \theta\left|\psi_{\text {anti }}\right\rangle\left\langle\psi_{\text {anti }}\left|+\cos ^{2} \theta\right| \psi_{\text {sym }}\right\rangle\left\langle\psi_{\text {sym }}\right|
$$

We quantify the accuracy of the reconstruction in terms of the fidelity [40,41]: $\mathscr{F}=$ $\left\langle\psi\left|\hat{\rho}_{\text {rec }}\right| \psi\right\rangle$, which for this example reads

$$
\mathscr{F}=\frac{1}{4}[3+\cos (4 \theta)],
$$

so it depends only on the single parameter $\theta$ that determines the projection onto the symmetric and antisymmetric subspaces, respectively. The minimum fidelity $\mathscr{F}=1 / 2$ corresponds to the case when the subspaces have the same weight, whereas for states in the completely symmetric or antisymmetric subspace, the reconstruction is exact. 
Our next example corresponds to an arbitrary pure three-qubit state, which can be written as

$$
|\psi\rangle=\sin \theta\left|\psi_{\mathrm{sym}}\right\rangle+e^{i \beta} \cos \theta \sin \alpha\left|\psi_{1}\right\rangle+e^{i \gamma} \cos \theta \cos \alpha\left|\psi_{2}\right\rangle,
$$

with $0 \leq \theta, \alpha \leq \pi / 2$ and $0 \leq \beta, \gamma<2 \pi .\left|\psi_{\text {sym }}\right\rangle$ is a state in the four-dimensional symmetric subspace, whereas $\left|\psi_{1}\right\rangle$ and $\left|\psi_{2}\right\rangle$ are states in SU(2)-irreducible twodimensional subspaces. In the computational basis, they are:

$$
\begin{aligned}
& \left|\psi_{\mathrm{sym}}\right\rangle=\sin \theta_{1}|000\rangle+e^{i \beta_{1}} \cos \theta_{1} \sin \alpha_{1} \frac{|100\rangle+|010\rangle+|001\rangle}{\sqrt{3}} \\
& +e^{i \gamma_{1}} \cos \theta_{1} \sin \alpha_{1} \sin \alpha_{2} \frac{|110\rangle+|101\rangle+|011\rangle}{\sqrt{3}} \\
& +e^{i \gamma_{2}} \cos \theta_{1} \cos \alpha_{1} \cos \alpha_{2}|111\rangle, \\
& \left|\psi_{1}\right\rangle=\sin \theta_{2} \frac{2|100\rangle-|010\rangle-|001\rangle}{\sqrt{6}}+e^{i \beta_{2}} \cos \theta_{2} \frac{|101\rangle-2|011\rangle+|110\rangle}{\sqrt{6}} \text {, } \\
& \left|\psi_{2}\right\rangle=\sin \theta_{3} \frac{|001\rangle-|010\rangle}{\sqrt{2}}+e^{i \beta_{3}} \cos \theta_{3} \frac{|101\rangle-|110\rangle}{\sqrt{2}} .
\end{aligned}
$$

The reconstructed density matrix is a mixed state, unless $|\psi\rangle$ is in the symmetric subspace. In particular, the blocks corresponding to two-dimensional SU(2)-irreducible subspaces have the same form; viz,

$$
\hat{\rho}_{2}=\left(\begin{array}{cc}
\sin ^{2} \alpha \sin ^{2} \theta_{2}+\cos ^{2} \alpha \sin ^{2} \theta_{3} & c_{2} \\
c_{2}^{*} & \cos ^{2} \alpha \cos ^{2} \theta_{3}+\cos ^{2} \theta_{2} \sin ^{2} \alpha
\end{array}\right),
$$

where $c_{2}=\frac{1}{2}\left[e^{-i \beta_{2}} \sin ^{2} \alpha \sin \left(2 \theta_{2}\right)+e^{-i \beta_{3}} \cos ^{2} \alpha \sin \left(2 \theta_{3}\right)\right]$ depends on the parameters $\alpha$ and $\theta$ 's describing the contributions from the three irreducible subspaces $\mathscr{S}_{\text {sym }}$, $\mathscr{S}_{1}$, and $\mathscr{S}_{2}$ in (25). Thus, the reconstructed density matrix has the form

$$
\hat{\rho}_{\text {rec }}=\sin ^{2} \theta\left|\psi_{\text {sym }}\right\rangle\left\langle\psi_{\text {sym }}\right| \oplus \frac{\cos ^{2} \theta}{2} \hat{\rho}_{2} \oplus \frac{\cos ^{2} \theta}{2} \hat{\rho}_{2} .
$$

By averaging over the phases $\theta_{2}, \beta_{2}$ and $\theta_{3}, \beta_{3}$ that parameterize the states in the nonsymmetric irreducible subspaces $\mathscr{S}_{1}$ and $\mathscr{S}_{2}$, we get the average fidelity that determines the distribution between the SU(2)-irreducible subspaces:

$$
\overline{\mathscr{F}}=\sin ^{2} \theta+\left[\frac{3}{4}+\frac{1}{4} \cos ^{2}(2 \alpha)\right] \cos ^{2} \theta \text {. }
$$

The minimum $\overline{\mathscr{F}}_{\text {min }}=3 / 4$ corresponds to the situation $|\psi\rangle=\left(\left|\psi_{1}\right\rangle+\left|\psi_{2}\right\rangle\right) / \sqrt{2}$ ( $\alpha=\pi / 4, \theta=0$ ) when the state is homogeneously distributed between not completely symmetric subspaces $\mathscr{S}_{1}$ and $\mathscr{S}_{2}$. The maximum fidelity is reached for symmetric states, $|\psi\rangle=\left|\psi_{\text {sym }}\right\rangle$. 


\section{Symmetric overcomplete tomography: canonical projection}

The outstanding case of fully symmetric (Dicke) states [42] deserves special attention as they are widely used in numerous applications (see, e.g. [43-45]) and, in addition, they are efficiently generated in the laboratory [46-49]. For Dicke states, the reconstruction (19) is exact, but requires $O\left(N^{3}\right)$ measurements of collective operators, while the density matrix contains at most $N^{2}+2 N$ independent parameters. Obviously, not all such collective measurements are independent. This redundancy can be fixed by representing the reconstructed density matrix via rank-one projectors.

For a fully symmetric density matrix, it follows from (5) that

$$
\begin{aligned}
\hat{\rho}_{\text {sym }} & =\hat{\Pi}_{\text {sym }}\left(\sum_{\alpha, \beta \in \mathbb{Z}_{2}^{N}} Q_{\rho_{\text {sym }}}(\alpha, \beta) \hat{\Delta}^{(1)}(\alpha, \beta)\right) \hat{\Pi}_{\text {sym }} \\
& =\sum_{\alpha, \beta \in \mathbb{Z}_{2}^{N}} \operatorname{Tr}\left[\hat{\rho}_{\text {sym }} \hat{\Delta}_{\text {sym }}^{(-1)}(\alpha, \beta)\right] \hat{\Delta}_{\text {sym }}^{(1)},
\end{aligned}
$$

with $\hat{\Delta}_{\text {sym }}^{( \pm 1)}(\alpha, \beta)=\hat{\Pi}_{\text {sym }} \hat{\Delta}^{( \pm 1)}(\alpha, \beta) \hat{\Pi}_{\text {sym }}$ and $\hat{\Pi}_{\text {sym }}=\sum_{\ell=0}^{N}|\ell, N\rangle\langle\ell, N|$ is the projection onto the Dicke subspace $\{|\ell, N\rangle: \ell=0, \ldots, N\}$ of $N$ qubits. It is shown in Appendix C.1 that $\hat{\Delta}_{\text {sym }}^{(-1)}(\alpha, \beta)$ is a symmetric function and actually it is a rank-one tensor

$$
\hat{\Delta}_{\mathrm{sym}}^{(-1)}(\alpha, \beta)=\left|\Psi_{h(\alpha), h(\beta), h(\alpha+\beta)}\right\rangle\left\langle\Psi_{h(\alpha), h(\beta), h(\alpha+\beta)}\right| .
$$

The unnormalized states $\left|\Psi_{h(\alpha), h(\beta), h(\alpha+\beta)}\right\rangle$ have the following expansion in the Dicke basis

$$
\left|\Psi_{h(\alpha), h(\beta), h(\alpha+\beta)}\right\rangle=\frac{1}{\left(1+|\xi|^{2}\right)^{N / 2}} \sum_{\ell=0}^{N}\left(\begin{array}{l}
N \\
\ell
\end{array}\right)^{-1 / 2} \psi_{\ell}(h(\alpha), h(\beta), h(\alpha+\beta) ; \xi)|\ell, N\rangle,
$$

$\psi_{\ell}(h(\alpha), h(\beta), h(\alpha+\beta) ; \xi)$ being a discrete function discussed in Appendix B.3.

The operators (31) form an informationally complete POVM

$$
\sum_{m, n, k} N_{m n k}^{2} R_{m n k}\left|\hat{\Psi}_{m n k}\right\rangle\left\langle\hat{\Psi}_{m n k}\right|=2^{N} \hat{\Pi}_{\text {sym }}
$$

where $\left|\hat{\Psi}_{m n k}\right\rangle=N_{m n k}^{-1}\left|\Psi_{m n k}\right\rangle$ and

$$
N_{m n k}^{2}=\frac{1}{\left(1+|\xi|^{2}\right)^{N}} \sum_{\ell=0}^{N}\left(\begin{array}{l}
N \\
\ell
\end{array}\right)^{-1}\left|\psi_{\ell}(m, n, k ; \xi)\right|^{2}
$$


For a given $(N+1)$-dimensional Dicke subspace, there are only $N$ different normalization factors $N_{m n k}$.

In terms of the projection of $\hat{\Delta}_{\mathrm{sym}}^{(1)}(\alpha, \beta)$, in Appendix C. 1 we arrive at the compact result

$$
\hat{\rho}_{\text {sym }}=\sum_{m, n, k} p_{m n k} R_{m n k} \hat{K}_{m n k}
$$

where $p_{m n k}=\left\langle\hat{\Psi}_{m n k}\left|\hat{\rho}_{\text {sym }}\right| \hat{\Psi}_{m n k}\right\rangle$ and

$$
\hat{K}_{m n k}=2^{-2 N} \sum_{m^{\prime}, n,^{\prime} k^{\prime}} 3^{\left(m^{\prime}+n^{\prime}+k^{\prime}\right) / 4} i^{\left(k^{\prime}-m^{\prime}-n^{\prime}\right) / 2} g_{m^{\prime} n^{\prime} k^{\prime}}(m, n, k) \hat{A}_{m^{\prime} n^{\prime} k^{\prime}}
$$

where $\hat{A}_{m n k}$ are also given in Appendix C.1.

In this protocol, the total number of projections (31) required for reconstruction of symmetric states is $\left(\begin{array}{c}N+3 \\ 3\end{array}\right)=(N+1)(N+2)(N+3) / 6$. However, it immediately follows from (35) that the probabilities $p_{m n k}$ are not linearly independent as they satisfy the conditions

$$
p_{m^{\prime} n^{\prime} k^{\prime}}=\sum_{m, n, k} p_{m n k} \omega_{m n k}^{m^{\prime} n^{\prime} k^{\prime}}
$$

where $\omega_{m n k}^{m^{\prime} n^{\prime} k^{\prime}}=R_{m n k}\left\langle\Psi_{m^{\prime} n^{\prime} k^{\prime}}\left|\hat{K}_{m n k}\right| \Psi_{m^{\prime} n^{\prime} k^{\prime}}\right\rangle$ and

$$
\begin{aligned}
\omega_{m n k}^{m^{\prime} n^{\prime} k^{\prime}}= & \frac{R_{m n k}}{\left(1+|\xi|^{2}\right)^{N}} \sum_{\ell, \ell^{\prime}=0}^{N}\left(\begin{array}{l}
N \\
\ell
\end{array}\right)^{-1 / 2}\left(\begin{array}{l}
N \\
\ell^{\prime}
\end{array}\right)^{-1 / 2} \\
& \times \psi_{\ell}(m, n, k ; \xi) \psi_{\ell^{\prime}}^{*}(m, n, k ; \xi) f_{\ell \ell^{\prime}}\left(m^{\prime}, k^{\prime}, n^{\prime}\right)
\end{aligned}
$$

These restrictions can be represented in a matrix form

$$
(\hat{\Omega}-1) \mathbf{p}=0
$$

where $\mathbf{p}$ is the $\left(\begin{array}{c}N+3 \\ 3\end{array}\right)$-dimensional probability vector and $\hat{\Omega}$ is an appropriately arranged matrix (38). We have numerically found that the rank of the matrix $(\hat{\Omega}-1)$ is $N\left(N^{2}-\right.$ $1) / 6$. Then, taking into account that the probabilities also satisfy the normalization condition $\operatorname{Tr}\left(\hat{\rho}_{\text {sym }}\right)=1$, we obtain that only $N^{2}+2 N$ projections are needed for the reconstruction of fully symmetric states.

\section{Concluding remarks}

In short, we have proposed a tomographic protocol based on measuring $\left(\begin{array}{c}N+3 \\ 3\end{array}\right)=$ $(N+1)(N+2)(N+3) / 6$ expectation values of collective operators. The advantage 
of the present approach with respect to previously discussed (and experimentally verified) methods is given by the explicit expressions (19) for the reconstructed density matrix from experimental data. In addition, we have shown that restricting ourselves to fully symmetric states, the tomographic protocol is reduced to projections from an overcomplete set of pure states (32), which still allows to obtain an explicit reconstruction expression (35). Such a set of states has been worked out from the first principles of state reconstruction in an $2^{N}$-dimensional Hilbert space.

Acknowledgements Open access funding provided by Max Planck Society. This work is partially supported by the Grant 254127 of CONACyT (Mexico). L. L. S. S. acknowledges the support of the Spanish MINECO (Grant FIS2015-67963-P).

Open Access This article is distributed under the terms of the Creative Commons Attribution 4.0 International License (http://creativecommons.org/licenses/by/4.0/), which permits unrestricted use, distribution, and reproduction in any medium, provided you give appropriate credit to the original author(s) and the source, provide a link to the Creative Commons license, and indicate if changes were made.

\section{A Properties of the symmetric operators $\hat{F}_{\text {mnk }}$}

The operators $\hat{F}_{m n k}$ can be expressed in terms of a special discrete function. Taking into account the action of the monomials $\hat{Z}_{\alpha} \hat{X}_{\beta}$ on the computational basis states $\left\{|\kappa\rangle: \kappa \in \mathbb{Z}_{2}^{N}\right\}$

$$
\hat{Z}_{\alpha}|\kappa\rangle=(-1)^{\alpha \kappa}|\kappa\rangle, \quad \hat{X}_{\beta}|\kappa\rangle=|\kappa+\beta\rangle,
$$

we immediately obtain for the matrix elements

$$
\left\langle\delta\left|\hat{F}_{m n k}\right| \gamma\right\rangle=(-i)^{\frac{1}{2}(m+n-k)} \delta_{n, h(\delta+\gamma)} f_{m k}(h(\delta), h(\gamma), h(\gamma+\delta)),
$$

with

$$
f_{m k}(h(\delta), h(\gamma), h(\gamma+\delta))=\sum_{\mu \in \mathbb{Z}_{2}^{N}} \delta_{h(\mu), m} \delta_{h(\mu+\gamma+\delta), k}(-1)^{\mu \delta} .
$$

The function $f_{m k}(h(\delta), h(\gamma), h(\gamma+\delta))$ will be further analyzed below.

By taking into account that $\operatorname{Tr}\left(\hat{Z}_{\mu} \hat{X}_{\lambda} \hat{Z}_{\mu^{\prime}} \hat{X}_{\lambda^{\prime}}\right)=2^{N}(-1)^{\lambda \mu^{\prime}} \delta_{\mu, \mu^{\prime}} \delta_{\lambda, \lambda^{\prime}}$, we get

$$
\begin{aligned}
\operatorname{Tr}\left(\hat{F}_{m n k} \hat{F}_{m^{\prime} n^{\prime} k^{\prime}}\right) & =2^{N} \delta_{m, m^{\prime}} \delta_{n, n^{\prime}} \delta_{k, k^{\prime}} \sum_{\mu, \lambda \in \mathbb{Z}_{2}^{N}} \delta_{h(\mu), m} \delta_{h(\lambda), n} \delta_{h(\mu+\lambda), k} \\
& =2^{N} R_{m n k} \delta_{m, m^{\prime}} \delta_{n, n^{\prime}} \delta_{k, k^{\prime}}
\end{aligned}
$$

which shows the orthogonality used in the paper. 


\section{B Special functions}

In this Appendix we discuss some relevant properties of the functions used in the derivation of our results.

\section{B.1 Function $g_{m n k}$}

The discrete function (18)

$$
g_{m n k}(h(\mu), h(\lambda), h(\mu+\lambda))=\sum_{\alpha, \beta \in \mathbb{Z}_{2}^{N}}(-1)^{\mu \beta+\lambda \alpha} \delta_{h(\alpha), m} \delta_{h(\beta), n} \delta_{h(\alpha+\beta), k}
$$

can be represented in the integral form

$$
\begin{aligned}
& g_{m n k}(h(\mu), h(\lambda), h(\mu+\lambda)) \\
&= \frac{1}{(2 \pi)^{3}} \oint \oint \oint \frac{d \omega_{1} d \omega_{2} d \omega_{3}}{\omega_{1}^{m+1} \omega_{2}^{n+1} \omega_{3}^{k+1}}\left(1+\omega_{1} \omega_{2}+\omega_{1} \omega_{3}+\omega_{2} \omega_{3}\right)^{N-\frac{1}{2}[h(\delta)+h(\gamma)+h(\delta+\gamma)]} \\
& \quad \times\left[1-\omega_{1} \omega_{2}-\omega_{1} \omega_{3}+\omega_{2} \omega_{3}\right]^{\frac{1}{2}[-h(\mu)+h(\lambda)+h(\mu+\lambda)]} \\
& \quad \times\left[1-\omega_{1} \omega_{2}+\omega_{1} \omega_{3}-\omega_{2} \omega_{3}\right]^{\frac{1}{2}[h(\mu)-h(\lambda)+h(\mu+\lambda)]} \\
& \quad \times\left[1+\omega_{1} \omega_{2}-\omega_{1} \omega_{3}-\omega_{2} \omega_{3}\right]^{\frac{1}{2}[h(\mu)+h(\lambda)-h(\mu+\lambda)]}
\end{aligned}
$$

where we have used the following representation of the Kronecker delta function

$$
\sum_{\kappa \in \mathbb{Z}_{2}^{N}} \delta_{h(\kappa), m}=\frac{1}{2 \pi} \int_{0}^{2 \pi} d x e^{-i x m} \prod_{i} \sum_{\kappa_{i}} e^{i x \kappa_{i}}
$$

The above integrals can be easily computed, leading to a quite cumbersome expression in terms of finite sums:

$$
\begin{aligned}
g_{m n k} & (h(\mu), h(\lambda), h(\mu+\lambda))=\sum_{j_{1}, \ldots, j_{10}}(-1)^{j_{3}+j_{4}+j_{6}+j_{5}-j_{1}-j_{7}} \delta_{k, n+m-2 j_{1}-2 j_{5}-2 j_{9}-2 j_{10}} \\
= & \left(\begin{array}{l}
j_{2} \\
j_{6}
\end{array}\right)\left(\begin{array}{l}
j_{3} \\
j_{7}
\end{array}\right)\left(\begin{array}{l}
j_{4} \\
j_{8}
\end{array}\right)\left(\begin{array}{c}
j_{6} \\
j_{10}
\end{array}\right)\left(\begin{array}{c}
j_{7} \\
j_{5}
\end{array}\right)\left(\begin{array}{c}
j_{8} \\
j_{1}
\end{array}\right)\left(\begin{array}{c}
N-\frac{1}{2}(h(\mu)+h(\lambda)+h(\mu+\lambda)) \\
n-\left(j_{1}+j_{2}+j_{3}+j_{4}+j_{5}+j_{9}+j_{10}-m\right)
\end{array}\right) \\
& \times\left(\begin{array}{c}
\frac{1}{2}(-h(\mu)+h(\lambda)+h(\mu+\lambda)) \\
j_{2}
\end{array}\right)\left(\begin{array}{c}
\frac{1}{2}(h(\mu)-h(\lambda)+h(\mu+\lambda)) \\
j_{3}
\end{array}\right) \\
& \times\left(\begin{array}{c}
\frac{1}{2}(h(\mu)+h(\lambda)-h(\mu+\lambda)) \\
j_{4}
\end{array}\right) \\
& \times\left(\begin{array}{c}
n-\left(j_{1}+j_{2}+j_{3}+j_{4}+j_{5}+j_{9}+j_{10}-m\right) \\
m-\left(j_{6}+j_{7}+j_{8}\right)
\end{array}\right)\left(\begin{array}{c}
m-\left(j_{6}+j_{7}+j_{8}\right) \\
j_{9}
\end{array}\right) .
\end{aligned}
$$


They satisfy the following dual orthogonality relations

$$
\begin{aligned}
& \sum_{m^{\prime}, n^{\prime}, k^{\prime}} g_{m n k}\left(m^{\prime}, n^{\prime}, k^{\prime}\right) g_{m^{\prime \prime} n^{\prime \prime} k^{\prime \prime}}\left(m^{\prime}, n^{\prime}, k^{\prime}\right) R_{m^{\prime} n^{\prime} k^{\prime}}=2^{2 N} R_{m n k} \delta_{m, m^{\prime \prime}} \delta_{n, n^{\prime \prime}} \delta_{k, k^{\prime \prime}}, \\
& \sum_{m^{\prime}, n^{\prime}, k^{\prime}} g_{m^{\prime} n^{\prime} k^{\prime}}(m, n, k) g_{m^{\prime} n^{\prime} k^{\prime}}\left(m^{\prime \prime}, n^{\prime \prime}, k^{\prime \prime}\right) R_{m^{\prime} n^{\prime} k^{\prime}}^{-1}=2^{2 N} R_{m n k}^{-1} \delta_{m, m^{\prime \prime}} \delta_{n, n^{\prime \prime}} \delta_{k, k^{\prime \prime}} .
\end{aligned}
$$

\section{B.2 Function $f_{m k}$}

Following a similar procedure, we can represent the function (42) in the integral form,

$$
\begin{aligned}
& f_{m k}(h(\delta), h(\gamma), h(\gamma+\delta))=\frac{1}{(2 \pi)^{2}} \oint \oint \frac{d \omega_{1} d \omega_{2}}{\omega_{1}^{m+1} \omega_{2}^{k+1}}\left(1+\omega_{1} \omega_{2}\right)^{N-\frac{1}{2}[h(\delta)+h(\gamma)+h(\delta+\gamma)]} \\
& \quad \times\left(\omega_{1}+\omega_{2}\right)^{\frac{1}{2}[h(\gamma)-h(\delta)+h(\delta+\gamma)]}\left(\omega_{2}-\omega_{1}\right)^{\frac{1}{2}[h(\delta)-h(\gamma)+h(\delta+\gamma)]}\left(1-\omega_{1} \omega_{2}\right)^{\frac{1}{2}[h(\delta)+h(\gamma)-h(\delta+\gamma)]} .
\end{aligned}
$$

Computing the integral (49) and rearranging the corresponding sums of binomial coefficients, we obtain

$$
\begin{aligned}
f_{m k}( & h(\delta), h(\gamma), h(\gamma+\delta))=(-1)^{m}\left(\begin{array}{c}
\frac{1}{2}[h(\delta)+h(\gamma)-n] \\
\frac{1}{2}(m-n+k)
\end{array}\right)\left(\begin{array}{c}
\frac{1}{2}[h(\delta)-h(\gamma)+n] \\
\frac{1}{2}(m+n-k)
\end{array}\right) \\
& \delta_{\frac{1}{2}(m+n-k) \in \mathbb{Z}} \delta_{\frac{1}{2}(m-n+k) \in \mathbb{Z}} \\
& \times{ }_{2} F_{1}\left(\frac{h(\delta)+h(\gamma)+n}{2}-N,-\frac{m-n+k}{2}, 1+\frac{h(\delta)+h(\gamma)-m-k}{2} ;-1\right) \\
& \times{ }_{2} F_{1}\left(\frac{-h(\delta)+h(\gamma)+n}{2},-\frac{m+n-k}{2}, 1+\frac{h(\delta)-h(\gamma)-m+k}{2} ;-1\right),(50)
\end{aligned}
$$

where ${ }_{2} F_{1}$ is the hypergeometric function. It is worth noting that $f_{m k}(h(\delta), h(\gamma), h(\gamma+$ $\delta))$ can be obtained by a reduction from $g_{m n k}(h(\delta), h(\gamma), h(\gamma+\delta))$.

\section{B.3 Function $\psi_{\ell}$}

The function $\psi_{\ell}$ is defined as

$$
\psi_{\ell}(h(\alpha), h(\beta), h(\alpha+\beta) ; \xi)=\sum_{\kappa \in \mathbb{Z}_{2}^{N}} \xi^{h(\kappa+\beta)}(-1)^{\alpha \kappa} \delta_{h(\kappa), \ell}
$$

and it can be recast as

$$
\psi_{\ell}(h(\alpha), h(\beta), h(\alpha+\beta), \xi)=\xi^{h(\beta)} \sum_{\kappa \in \mathbb{Z}_{2}^{N}} \xi^{h(\kappa)-2 \sum_{i} \beta_{i} \kappa_{i}}(-1)^{\alpha \kappa} \delta_{h(\kappa), \ell}
$$




$$
\begin{aligned}
= & \xi^{h(\beta)} \int \frac{d \omega}{\omega^{1+\ell}} \prod_{i}^{N} \sum_{\kappa_{i}=0}^{1} \xi^{\kappa_{i}-2 \beta_{i} \kappa_{i}}(-1)^{\alpha_{i} \kappa_{i}} \omega^{\kappa_{i}} \\
= & \xi^{h(\beta)} \int \frac{d \omega}{\omega^{1+\ell}}(1+\xi \omega)^{N-\frac{1}{2}[h(\alpha)+h(\beta)+h(\alpha+\beta)]}(1-\xi \omega)^{\frac{1}{2}[h(\alpha)-h(\beta)+h(\alpha+\beta)]} \\
& \times\left(1+\xi^{-1} \omega\right)^{\frac{1}{2}[-h(\alpha)+h(\beta)+h(\alpha+\beta)]}\left(1-\xi^{-1} \omega\right)^{\frac{1}{2}[h(\alpha)+h(\beta)-h(\alpha+\beta)]}
\end{aligned}
$$

which leads to the following expression in terms of finite sums

$$
\begin{aligned}
\psi_{\ell} & (h(\alpha), h(\beta), h(\alpha+\beta), \xi) \\
= & \xi^{l+h(\beta)} \sum_{j_{2}, j_{3}, j_{4}}(-1)^{j_{3}+j_{4}} \xi^{-2\left(j_{2}+j_{4}\right)}\left(\begin{array}{c}
N-\frac{1}{2}[h(\alpha)+h(\beta)+h(\alpha+\beta)] \\
l-j_{2}-j_{3}-j_{4}
\end{array}\right) \\
& \times\left(\begin{array}{c}
\frac{1}{2}[-h(\alpha)+h(\beta)+h(\alpha+\beta)] \\
j_{2}
\end{array}\right)\left(\begin{array}{c}
\frac{1}{2}[h(\alpha)-h(\beta)+h(\alpha+\beta)] \\
j_{3}
\end{array}\right) \\
& \times\left(\begin{array}{c}
\frac{1}{2}[h(\alpha)+h(\beta)-h(\alpha+\beta)] \\
j_{4}
\end{array}\right) .
\end{aligned}
$$

\section{Canonical projection}

In this section we find projections of the kernels $\hat{\Delta}^{( \pm 1)}(\alpha, \beta)$ onto the Dicke subspace.

\section{C.1 Projection of $\hat{\Delta}^{(-1)}(\alpha, \beta)$}

Taking into account the representation of the Dicke states in the logical basis,

$$
|\ell, N\rangle=\frac{1}{\sqrt{\left(\begin{array}{c}
N \\
\ell
\end{array}\right)}} \sum_{\substack{\kappa \in \mathbb{Z}_{2}^{N} \\
h(\kappa)=\ell}}|\kappa\rangle
$$

we obtain

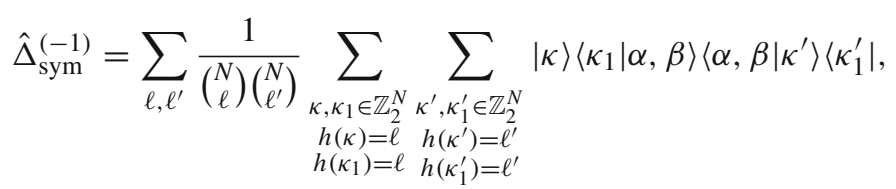


where the discrete coherent states $|\alpha, \beta\rangle$ are defined in (7). Using the expansion of the fiducial state (3) in the logical basis

$$
|\xi\rangle=\frac{1}{\left(1+|\xi|^{2}\right)^{N / 2}} \sum_{\kappa \in \mathbb{Z}_{n}^{2}} \xi^{h(\kappa)}|\kappa\rangle,
$$

we get

$$
\langle\kappa \mid \alpha, \beta\rangle=\frac{1}{\left(1+|\xi|^{2}\right)^{N / 2}} \xi^{h(\kappa+\beta)}(-1)^{\alpha \kappa} .
$$

By substituting (58) into (56), we arrive at (31).

The operator $\hat{\Delta}_{\mathrm{sym}}^{(1)}(\alpha, \beta)$ can also be expressed as

$$
\begin{aligned}
\hat{\Delta}_{\mathrm{sym}}^{(1)}(h(\alpha), h(\beta), h(\alpha+\beta))= & \frac{1}{2^{2 N}} \sum_{m, n, k} 3^{(m+n+k) / 4} i^{(k-m-n) / 2} \\
& g_{m n k}(h(\alpha), h(\beta), h(\alpha+\beta)) \hat{A}_{m n k},
\end{aligned}
$$

where $g_{m n k}(h(\alpha), h(\beta), h(\alpha+\beta))$ is defined in (18), and the matrix elements of the operators $\hat{A}_{m n k}$ in the Dicke basis are

$$
\left\langle\ell^{\prime}, N\left|\hat{A}_{m n k}\right| \ell, N\right\rangle=\frac{1}{\sqrt{\left(\begin{array}{c}
N \\
\ell
\end{array}\right)\left(\begin{array}{l}
N \\
\ell^{\prime}
\end{array}\right)}} f_{\ell \ell^{\prime}}(m, k, n) .
$$

Finally, using the summation rule

$$
\sum_{\alpha, \beta \in \mathbb{Z}_{2}^{N}} f(\alpha, \beta)=\sum_{m, n, k} \sum_{\alpha, \beta \in \mathbb{Z}_{2}^{N}} \delta_{h(\alpha), m} \delta_{h(\beta), n} \delta_{h(\alpha+\beta), k} f(\alpha, \beta),
$$

we get the explicit expression (35).

\section{C.2 Projection of monomials $\hat{\Pi}_{\text {sym }} \hat{Z}_{\alpha} \hat{x}_{\beta} \hat{\Pi}_{\text {sym }}$}

It follows immediately from (40) that the matrix elements of the monomial $\hat{Z}_{\alpha} \hat{X}_{\beta}$ in the Dicke basis (55) have the form

$$
\begin{aligned}
\left\langle\ell, N\left|\hat{\Pi}_{\mathrm{sym}} \hat{Z}_{\alpha} \hat{X}_{\beta} \hat{\Pi}_{\mathrm{sym}}\right| \ell^{\prime}, N\right\rangle & =\frac{1}{\sqrt{\left(\begin{array}{c}
N \\
\ell
\end{array}\right)\left(\begin{array}{c}
N \\
\ell^{\prime}
\end{array}\right.}} \sum_{\mu \in \mathbb{Z}_{2}^{N}}(-1)^{\alpha \mu} \delta_{h(\mu), \ell} \delta_{h(\mu+\beta), \ell^{\prime}} \\
& =\frac{f_{\ell \ell^{\prime}}(h(\alpha), h(\alpha+\beta), h(\beta))}{\sqrt{\left(\begin{array}{c}
N \\
\ell
\end{array}\right)\left(\begin{array}{c}
N \\
\ell^{\prime}
\end{array}\right)}}
\end{aligned}
$$

where the function $f_{\ell \ell^{\prime}}$ is defined in (42). 


\section{References}

1. Lvovsky, A.I., Raymer, M.G.: Continuous-variable optical quantum-state tomography. Rev. Mod. Phys. 81(299-322), 299 (2009)

2. Paris, M.G.A., Řeháček, J. (eds.): Quantum State Estimation, vol. 649 Lecture Notes in Physics. Springer, Berlin (2004)

3. Wootters, W.K., Fields, B.D.: Optimal state-determination by mutually unbiased measurements. Ann. Phys. 191, 363-381 (1989)

4. Renes, J.M., Blume-Kohout, R., Scott, A.J., Caves, C.M.: Symmetric informationally complete quantum measurements. J. Math. Phys. 45, 2171-2180 (2004)

5. Lima, G., Neves, L., Guzmán, R., Gómez, E.S., Nogueira, W.A.T., Delgado, A., Vargas, A., Saavedra, C.: Experimental quantum tomography of photonic qudits via mutually unbiased basis. Opt. Express 19, 3542-3552 (2011)

6. Bent, N., Qassim, H., Tahir, A.A., Sych, D., Leuchs, G., Sánchez-Soto, L.L., Karimi, E., Boyd, R.W.: Experimental realization of quantum tomography of photonic qudits via symmetric informationally complete positive operator-valued measures. Phys. Rev. X 5, 041006 (2015)

7. Häffner, H., Hänsel, W., Roos, C.F., Benhelm, J., Chwalla, M., Körber, T., Rapol, U.D., Riebe, M., Schmidt, P.O., Becher, C., Gühne, O., Dür, W., Blatt, R.: Scalable multiparticle entanglement of trapped ions. Nature 438, 643-646 (2005)

8. Hou, Z., Zhong, H.-S., Tian, Y., Dong, D., Qi, B., Li, L., Wang, Y., Nori, F., Xiang, G.-Y., Li, C.-F., Guo, G.-C.: Full reconstruction of a 14-qubit state within four hours. New J. Phys. 18, 083036 (2016)

9. Gross, D., Liu, Y.K., Flammia, S.T., Becker, S., Eisert, J.: Quantum state tomography via compressed sensing. Phys. Rev. Lett. 105, 15040 (2010)

10. Flammia, S.T., Gross, D., Liu, Y.-K., Eisert, J.: Quantum tomography via compressed sensing: error bounds, sample complexity and efficient estimators. New J. Phys. 14, 095022 (2012)

11. Guta, M., Kypraios, T., Dryden, I.: Rank-based model selection for multiple ions quantum tomography. New J. Phys. 14, 105002 (2012)

12. Riofrío, C.A., Gross, D., Flammia, S.T., Monz, T., Nigg, D., Blatt, R., Eisert, J.: Experimental quantum compressed sensing for a seven-qubit system. Nat. Commun. 8, 15305 (2017)

13. Cramer, M., Plenio, M.B., Flammia, S.T., Somma, R., Gross, D., Bartlett, S.D., Landon-Cardinal, O., Poulin, D., Liu, Y.K.: Efficient quantum state tomography. Nat. Commun. 1, 149 (2010)

14. Baumgratz, T., Gross, D., Cramer, M., Plenio, M.B.: Scalable reconstruction of density matrices. Phys. Rev. Lett 111, 020401 (2013)

15. Landon-Cardinal, O., Poulin, D.: Practical learning method for multi-scale entangled states. New J. Phys. 14, 085004 (2012)

16. D’Ariano, G.M., Maccone, L., Paini, M.: Spin tomography. J. Opt. B 5, 77-84 (2003)

17. Tóth, G., Wieczorek, W., Gross, D., Krischek, R., Schwemmer, C., Weinfurter, H.: Permutationally invariant quantum tomography. Phys. Rev. Lett. 105, 20040 (2010)

18. Moroder, T., Hyllus, P., Tóth, G., Schwemmer, C., Niggebaum, A., Gaile, S., Gühne, O., Weinfurter, H.: Permutationally invariant state reconstruction. New J. Phys. 14, 105001 (2012)

19. Klimov, A.B., Björk, G., Sánchez-Soto, L.L.: Optimal quantum tomography of permutationally invariant qubits. Phys. Rev. A 87, 012109 (2013)

20. Schwemmer, C., Tóth, G., Niggebaum, A., Moroder, T., Gross, D., Gühne, O., Weinfurter, H.: Experimental comparison of efficient tomography schemes for a six-qubit state. Phys. Rev. Lett. 113, 040503 (2014)

21. French, S.R.D., Rickles, D.P.: Understanding permutation symmetry. In: Brading, K., Castellani, E. (eds.) Symmetries in Physics: Philosophical Reflections, pp. 212-238. Cambridge University Press, Cambridge (2003)

22. Inguscio, M., Fallani, L.: Atomic Physics: Precise Measurements and Ultracold Matter. Oxford University Press, Oxford (2015)

23. Chuang, I., Nielsen, M.: Quantum Computation and Quantum Information. Cambridge University Press, Cambridge (2000)

24. Björk, G., Klimov, A.B., Sánchez-Soto, L.L.: The discrete Winger function. Prog. Opt. 51, 469-516 (2008)

25. Muñoz, C., Klimov, A.B., Sánchez-Soto, L.L.: Symmetric discrete coherent states for $n$-qubits. J. Phys. A: Math. Theor. 45, 244014 (2012)

26. Schroek, F.E.: Quantum Mechanics on Phase Space. Kluwer, Dordrecht (1996) 
27. Zachos, C.K., Fairlie, D.B., Curtright, T.L. (eds.): Quantum Mechanics in Phase Space. World Scientific, Singapore (2005)

28. Galetti, D., Marchiolli, M.A.: Discrete coherent states and probability distributions in finitedimensional spaces. Ann. Phys. 249, 454-480 (1996)

29. Marchiolli, M.A., Ruzzi, M., Galetti, D.: Discrete squeezed states for finite-dimensional spaces. Phys. Rev. A 76, 032102 (2007)

30. Muñoz, C., Klimov, A.B., Sánchez-Soto, L.L.: Discrete coherent states for $n$ qubits. Int. J. Quantum Inf. 7, 17-25 (2009)

31. Klimov, A.B., Muñoz, C., Sánchez-Soto, L.L.: Discrete coherent and squeezed states of many-qudit systems. Phys. Rev. A 80, 043836 (2009)

32. Klimov, A.B., Romero, J.L., Björk, G., Sánchez-Soto, L.L.: Discrete phase-space structure of $n$-qubit mutually unbiased bases. Ann. Phys. 324, 53-72 (2009)

33. Prugovečki, E.: Information-theoretical aspects of quantum measurement. Int. J. Theor. Phys. 16, 321-331 (1977)

34. Busch, P., Lahti, P.J.: The determination of the past and the future of a physical system in quantum mechanics. Found. Phys. 19, 633-678 (1989)

35. Galetti, D., de Toledo Piza, A.F.R.: Discrete quantum phase spaces and the $\bmod n$ invariance. Physica A 186, 513-523 (1992)

36. Ruzzi, M., Marchiolli, M.A., Galetti, D.: Extended Cahill-Glauber formalism for finite-dimensional spaces: I. Fundamentals. J. Phys. A 38, 6239 (2005)

37. Montanaro, A.: Symmetric functions of qubits in an unknown basis. Phys. Rev. A 79, 062316 (2009)

38. Klimov, A.B., Muñoz, C.: Macroscopic features of quantum fluctuations $n$ qubit systems. Phys. Rev. A 89, 052130 (2014)

39. Gaeta, M., Muñoz, C., Klimov, A.B.: Gaussianity and localization of $n$-qubit states. Phys. Rev. A 93, 062107 (2016)

40. Uhlmann, A.: The transition probability in the state space of a -algebra. Rep. Math. Phys. 9, 273-279 (1976)

41. Jozsa, R.: Fidelity for mixed quantum states. J. Mod. Opt. 41, 2315-2323 (1994)

42. Dicke, R.: Coherence in spontaneous radiation processes. Phys. Rev. 93, 99 (1954)

43. Di, T., Muthukrishnan, A., Scully, M.O., Zubairy, M.S.: Quantum teleportation of an arbitrary superposition of atomic Dicke states. Phys. Rev. A 71, 062308 (2005)

44. Chiuri, A., Greganti, C., Paternostro, M., Vallone, G., Mataloni, P.: Experimental quantum networking protocols via four-qubit hyperentangled dicke states. Phys. Rev. Lett. 109, 173604 (2012)

45. Apellaniz, I., Lücke, B., Peise, J., Klempt, C., Tóth, G.: Detecting metrologically useful entanglement in the vicinity of Dicke states. New J. Phys. 17, 083027 (2015)

46. Duan, L.-M., Kimble, J.H.: Efficient engineering of multiatom entanglement through single-photon detections. Phys. Rev. Lett. 90, 253601 (2003)

47. Kiesel, N., Schmid, C., Tóth, G., Solano, E., Weinfurter, H.: Experimental observation of four-photon entangled Dicke state with high fidelity. Phys. Rev. Lett. 98, 063604 (2007)

48. Wieczorek, W., Krischek, R., Kiesel, N., Michelberger, P., Tóth, G., Weinfurter, H.: Experimental entanglement of a six-photon symmetric Dicke state. Phys. Rev. Lett. 103, 020504 (2009)

49. Prevedel, R., Cronenberg, G.M.S., Tame, M.S., Paternostro, M., Walther, P., Kim, M.S., Zeilinger, A.: Experimental realization of Dicke states of up to six qubits for multiparty quantum networking. Phys. Rev. Lett. 103, 020503 (2009) 